\title{
REVISÃO DE PECOPTERÍDEAS POLIMÓRFICAS DO NEOPALEOZÓICO DA AMÉRICA DO SUL
}

\author{
CARLOS EDUARDO LUCAS VIEIRA \\ Laboratório de Micropaleontologia, UNISINOS, Av. Unisinos, 950, 93022-000, São Leopoldo, RS, Brasil. \\ carlosev@unisinos.br \\ ROBERTO IANNUZZI \& MARGOT GUERRA-SOMMER \\ IG, UFRGS, Av. Bento Gonçalves, 9500, 91540-970, Porto Alegre, RS, Brasil.roberto.iannuzzi@ufrgs.br, \\ margot.sommer@ufrgs.br
}

\begin{abstract}
RESUMO - Pecopteris pedrasica e Asterotheca piatnitzkyi tiveram aqui suas diagnoses emendadas a fim de abranger a polimorfia de suas frondes e esclarecer as ambigüidades na classificação taxonômica das mesmas. Da análise desta polimorfia, foi possível estabelecer um modelo de desenvolvimento para estas frondes, em que a diferenciação ocorre segundo um gradiente topológico das porções proximais para as distais e das porções basiscópicas para as acroscópicas. Este modelo é de grande valia não só para o entendimento da ontogenia de frondes, mas também para a classificação de formas fósseis que porventura apresentem-se incompletas. Com a resolução taxonômica aqui empreendida, pode-se também estabelecer uma correlação mais efetiva entre as distintas associações pecopterídeas da América do Sul. Esta mesma resolução, permitiu constatar que $P$. pedrasica é a pecopterídea de maior distribuição espacial e temporal do Neopaleozóico sul-americano. Esta distribuição confere um indiscutível caráter euritópico a P. pedrasica, uma vez que os ambientes e as variações climáticas enfrentadas por esta forma nas regiões habitadas ao longo do intervalo de tempo vivido, foram muito diversos.
\end{abstract}

Palavras-chaves: Frondes polimórficas, Asterotheca piatnitzkyi, Pecopteris pedrasica, Permiano, América do Sul.

ABSTRACT - REVIEW OF POLYMORPHIC PECOPTERIDS FROM SOUTH AMERICA NEOPALEOZOIC. Pecopteris pedrasica and Asterotheca piatnitzkyi diagnosis were emended here in order to involve their polymorphic nature and to resolve their present taxonomic ambiguity. A model for frond development was established from analysis of that polymorphy where the differentiation occur according to a topologic gradient from proximal and basiscopic to distal and acroscopic portions. This model is useful both to the frond ontogeny understanding and the classification of those putative incomplete forms. The distinct South American pecopterid associations could be more closely correlated by the taxonomic resolution here proposed. This resolution permitted concludes that P. pedrasica is the pecopterid with the broadest spatial and temporal South America Neopaleozoic distribution. This distribution provides an indisputable eurytopic nature to $P$. pedrasica inasmuch the environment and climatic changes endured for this form have been too diversified.

Key words: Polymorphic fronds, Asterotheca piatnitzkyi, Pecopteris pedrasica, Permian, South America.

\section{INTRODUÇÃO}

A polimorfia é o fenômeno pelo qual um determinado trato biológico apresenta-se sob distintas formas em uma mesma espécie ou indivíduo. Ela ocorre por processos heteroblásticos, ou seja, pela mudança nas taxas de crescimento histológico do trato em questão. Estas mudanças podem ser tanto heterocrônicas (filogeneticamente determinadas), como ecofisiológicas (ambientalmente determinadas). Dada a natureza modular e independente do crescimento das plantas, polimorfias podem ocorrer repetidamente a qualquer momento e lugar, ou seja, tanto em um mesmo, quanto em diferentes estágios ontogenéticos de um indivíduo (Guerrant Jr., 1988; Kerstetter \& Poethig, 1998; Diggle, 1999; Tsukaya, 2002). Nas plantas com frondes, a polimorfia se expressa nas pinas e pínulas de um mesmo espécime ou espécie, geralmente obedecendo a um padrão topológico de diferenciação morfológica (Goliber et al., 1999). Em frondes fósseis, é comum que uma ou outra destas formas acabe preservada isoladamente das demais. Sob estas condições, pode-se facilmente tomar cada forma como uma espécie distinta.

Archangelsky \& De La Sota (1960) identificaram como tipos morfológicos de uma mesma fronde as seguintes morfoespécies de pecopterídeas: Pecopteris pedrasica Read, $P$. cambuhyensis Read e Asterotheca piatnitzkyi Frenguelli. Esta identificação, no entanto, não foi acompanhada de um esclarecimento sobre a classificação taxonômica dos espécimes envolvidos.

\section{Revisão das formas descritas}

O primeiro registro destas formas pecopterídeas foi feito por Feruglio (1933), ao estudar amostras provenientes de estratos da Formação La Golondrina (Permiano Inferior da bacia La Golondrina) na Argentina. As amostras eram compostas por espécimes pecopterídeos férteis e estéreis, ambos apresentando margens límbicas, lobuladas ou retas e classifcados, indistintamente, como Pecopteris sp. II. Exemplares estéreis com estas mesmas características foram encontrados por Read (1941) em estratos da Formação Rio 
Bonito (Permiano Inferior da bacia do Paraná) que propôs as morfoespécies Pecopteris cambuhyensis e P. pedrasica para classificar os espécimes que apresentavam, respectivamente, margens límbicas retas e margens lobuladas.

Posteriormente, Feruglio (1951) observou que havia uma nítida similaridade entre os espécimes que ele havia descrito previamente como Pecopteris sp. II e aqueles identificados como P. cambuhyensis e P. Paranaensis por Read (1941). Ressalvando-se o fato de que é $P$. pedrasica que apresenta margens lobuladas e não $P$. paranaensis, a semelhança observada foi extremamente pertinente, mas, a despeito disto, Feruglio optou por manter seus espécimes sob a designação de Pecopteris sp. II, em função de diferenças de tamanho pinular com $P$. cambuhyensis e $P$. paranaensis. Entretanto, estas mesmas diferenças existiam entre os espécimes incluidos em $P$. sp. II, o que denota uma ambigüidade na avaliação desta característica como caráter taxonômico.

Frenguelli (1953) corrigiu esta ambigüidade classificando $P$. sp. II (Feruglio, 1933, 1951) como P. cambuhyensis e $P$. pedrasica. A mesma classificação tiveram os novos espécimes pecopterídeos estéreis que ele encontrara também na Formação La Golondrina (bacia La Golondrina, Argentina), porém, aqueles encontrados em estágio fértil, foram então classificados como Asterotheca piatnitzkyi.

Examinando novas pecopterídeas provenientes da Formação La Golondrina, Archangelsky \& De La Sota (1960) concluíram que $P$. pedrasica e $P$. cambuhyensis eram formas diferentes de uma mesma fronde, cujo estágio fértil era idêntico a A. piatnitzkyi. Em outras palavras, foi demonstrado, pela primeira vez, o caráter polimórfico destas frondes. Com base nisto, elegeram $A$. piatnitzkyi como o nome representante desta pecopterídea polimórfica, incluindo em sinonímia as formas estéreis classificadas por Frenguelli (1953) como $P$. pedrasica e $P$. cambuhyensis.

Rösler (1972), com base em novos espécimes da Formação Rio Bonito, sugeriu o nome Asterotheca (Pecopteris) cambuhyensis a esta forma polimórfica, baseando-se na precedência que as espécies de Read (1941) tinham em relação às de Frenguelli (1953) e de Archangelsky \& De La Sota (1960). Esta alternativa taxonômica foi criticada por CazzuloKlepzig (1978), para quem o nome Pecopteris (Asterotheca) cambuhyensis parecia mais apropriado. Entretanto, ambos os nomes foram desconsiderados pela autora, que classificou seus espécimes estéreis como $P$. pedrasica.

Desde então, os espécimes pecopterídeos correspondentes as formas citadas aqui passaram a ser classificados ou como Asterotheca piatnitzkyi, ou como Pecopteris pedrasica (vide, Azcuy \& Césari, 1980; Rohn \& Rösler, 1986; Vieira et al., 2004). Embora a aceitação tácita destes nomes indique um acerto quanto ao reconhecimento da necessidade paleobotânica de distinção parataxonômica entre espécimes férteis e estéreis de uma mesma forma, as diagnoses originais de $P$. pedrasica e $A$. piatnitzkyi não contemplam a referida polimorfia destas pecopterídeas e não está claro se espécimes estéreis encontram-se classificados indistintamente como A piatnitzkyi.

O presente trabalho visa a fornecer diagnose e classificação apropriada a estas frondes conforme as normas do Código Internacional de Nomenclatura Botânica (Greuter et al., 2000), bem como discutir as implicações bioestratigráficas, paleoecológicas e paleogeográficas no cenário neopaleozóico sul-americano.

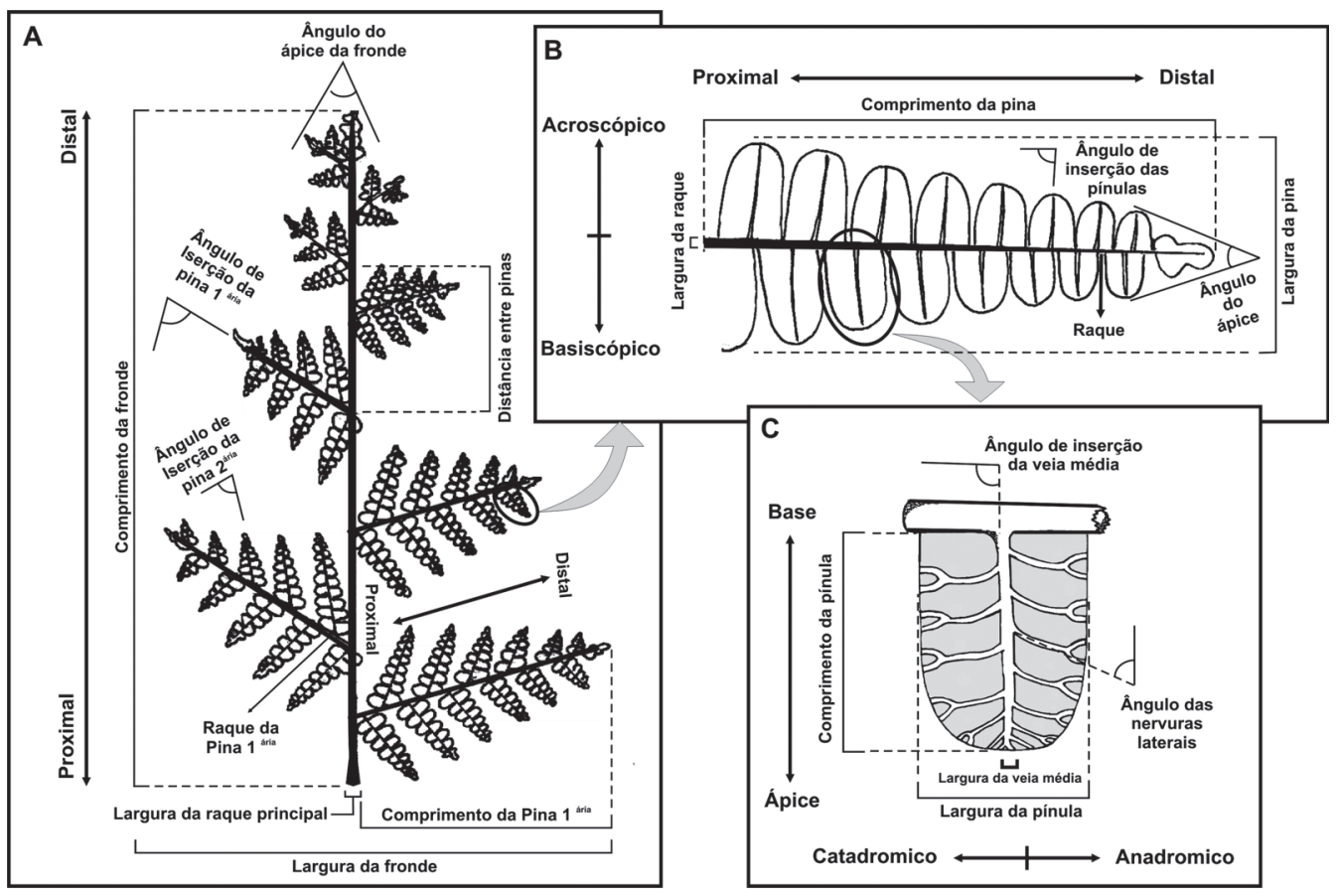

Figura 1. Parâmetros empregados na descrição de pteridófilas: A, fronde tripinada; B, detalhe da pina secundária destacada em A; C, detalhe de uma pínula pecopterídea destacada em B (adaptado de Rohn \& Rösler, 1986).

Figure 1. Parameters used in pteridophylla description: A, tripinnate frond; B, secondary pinna detail from A; C, pecopterid pinnule detail from B (adapted from Rohn \& Rösler, 1986). 


\section{MATERIAL E MÉTODOS}

O material descrito por Read (1941) como P. pedrasica e $P$. cambuhyensis, depositado na coleção de paleobotânica(USNM) do National Museum of Natural History (Washington DC), foi estudado a partir de fotografias detalhadas dos espécimes.

Os espécimes identificados por Cazzulo-Klepzig (1978) como P. pedrasica encontram-se na coleção de paleobotânica (MP-Pb) do Instituto de Geociências da Universidade Federal do Rio Grande do Sul (UFRGS), em Porto Alegre.

Os espécimes de A. piatnitzkyi descritos por Frenguelli (1953) e Azcuy \& Césari (1980) encontram-se depositados, respectivamente, na coleção de paleobotânica (LIL) do Museo Miguel Lillo, em San Miguel de Tucumán e na coleção de paleobotânica (BAFCPb) da Facultad de Ciencias da Universidad de Buenos Aires (UBA), em Buenos Aires.

Os espécimes descritos por Rösler (1972) como A. (P.) cambuhyensis encontram-se depositados na coleção de paleobotânica (GP/3T ou GP/3E) do Instituto de Geociências da Universidade de São Paulo (USP), em São Paulo.

Na descrição do material foram empregados os parâmetros arrolados por Rohn \& Rösler (1986), e adaptados no presente trabalho (Figura 1).

\section{RESULTADOS}

\section{Divisão PTERIDOPHYTA}

Família MARATTIACEAE

Gênero Asterotheca Presl, in Corda, 1845

Espécie-tipo. Asterotheca sternbergii (Goeppert) Presl, in Corda, 1845.

\section{Asterotheca piatnitzkyi Frenguelli (emend. Vieira et al.)}

(Figura 2; Tabela 1)

Pecopteris sp.2 Feruglio, Ann. R. Mus. Geol. di Bologna, 9:10, lam. 2, fig. 3; lam. 3, figs. 1-2, 1933

Asterotheca piatnitzkyi Frenguelli, Notas Mus. Eva Perón 16(99):291; lam. III-IV, 1953

Asterotheca piatnitzkyi. Archangelsky, Acta Geol. Lil., 2:35. figs. 12-4, 16, 1958

Asterotheca piatnitzkyi. Archangelsky \& De La Sota, Acta Geol. Lil., 3:93; lam. II, figs.7-13; lam. III, fig. 15, 1960

Asterotheca piatnitzkyi. Azcuy \& Césari, Bol. IG-USP,11:51. fig. 2; lam. 1, figs. 1-4, 1980

Lectótipo. LP 5617, col. paleobot. Mus. Hist. Nat. La Plata, Frenguelli, 1953, lam. 4, figs.1-2.

Material estudado. LIL 1119b, 1125, 1131b-2b, 1359b, 1408b, 1644, 1658, 1802-4, 1881a, 1882a, 1883a, 1884a, 1885a, 1886a, 2000a, 2001, 2002a, 2003a, 2004a, 2005-11a, 2012a, 2013a, 2014a, 2015, 2016a, 2017a, 2018-20, 2021a, 2022a, 2023a, 2024-28, 2029a, 2030a, 2031a, 2032a, 2033a, 2034, 2035a, 2036, 2037a, 2038a, 2039a, 2040a, 2041,2042a, 2043; LILNF3-1675,1677; NF5 - 1227b, 1228b, 1318b, 1354a, 1386b, 1401a, 1641, 1642, 1646, 1649, 1650, 1651, 1654b, 1656a, 1657a, 1660a, 1701-4, 1724, 1821a; NF6 - 1718, 1720, 1745; NFVII - 1643, 1647, 1648, 1721, 1724; NFb - 1757-9; NFc - 1805, 1810; NFd-1811(?), 1812-20; NFe-1440, 1601b, 1692, 1694, 1697,
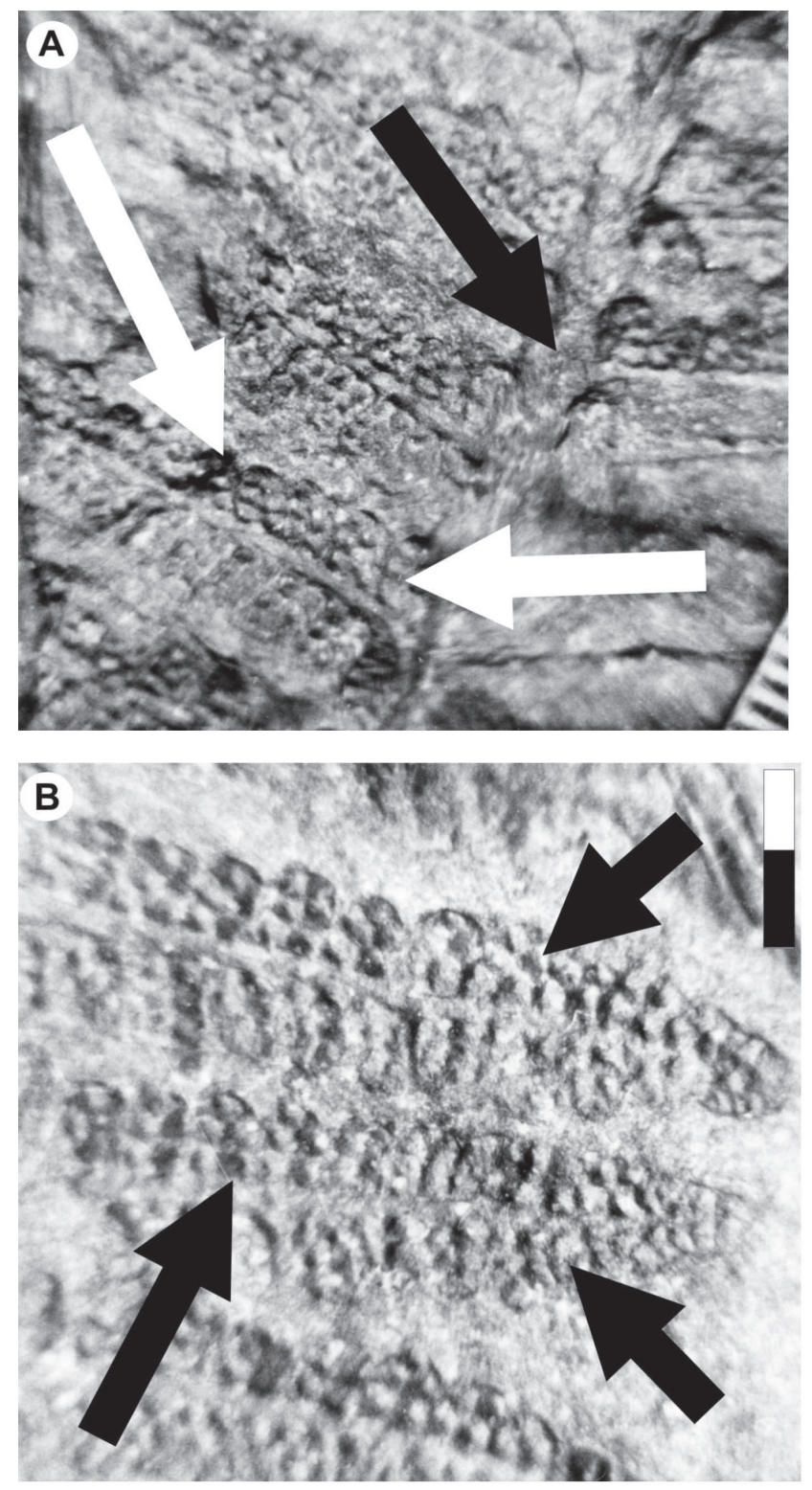

Figura 2. Asterotheca piatnitzkyi, LIL 2015: A, pina primária com pinas secundárias e pínulas indicando a raque da pina primária (seta preta) e sinângios rosetados (setas brancas) sobre as pínulas, escala em $\mathrm{mm}$; $\boldsymbol{B}$, detalhe de pinas secundárias com sinângios rosetados sobre as pínulas (setas pretas), escala $=6 \mathrm{~mm}$. Figure 2. Asterotheca piatnitzkyi, LIL 2015: A, primary pinna with visible secondary pinnae and pinnulae showing primary pinna rachis (black arrow) and stellar sinangia on the pinnulae (white arrow), scale inmm; $\mathbf{B}$, secondary pinnae with stellar sinangia on the pinnulae (black arrows), scale bar $=6 \mathrm{~mm}$.

$1698,1700,1705-10,1712-17,1733-44 ; \mathrm{NFg}-1661,1662,1664$, 1678-80, 1683, 1687-90. BAFCPb 10617, 10621, 10623-27, 1071823,10741-43, 10746, 10749, 10750, 10780.

Emmended diagnosis. Polymorphic frond at least tripinnate, lanceolate, main rachis $17 \mathrm{~mm}$ width in average. Lanceolate primary and secondary pinna, very close to each other, alternately arranged on axis at $90^{\circ}$ angles. Secondary pinna either like a lobulated or pinnatifid pinnule with $3 \mathrm{~cm}$ length and obtusely rounded apex when undifferentiated; truly pinnate and linear-laceolated when differentiated. Pinna 
rachis similar to a straight mid-vein with lateral veins unevenly dichotomized two or more times on their curved course to margin. Differentiated secondary pinna pinnate and linear-lanceolated in outline. Pinnule membranaceous, obliquely to right inserted by the entire base, alternate and contiguous, terminating in an obtusely rounded apex, nearly $12 \mathrm{~mm}$ long. Venation tenuous, straight mid-vein ascending near the apex and dichotomizing once; lateral veins arise alternately from the mid-vein and dichotomize 1-2 times on their curved course to the margin. Six to 11 pairs of circular sinangia parallel to the mid-vein with 5 or 6 (rarely 4) fusiform sporangia; monolete or pseudotrilete spores, slightly punctuated, quasilcevigate.

Diagnose emendada. Frondes polimórficas, no mínimo tripinadas, lanceoladas, raque principal com $17 \mathrm{~mm}$ de largura média. Pinas primárias e secundárias lanceoladas, bastante próximas entre si, alternadamente arranjadas sobre seus eixos, em ângulos perpendiculares. Pinas secundárias pinatífidas ou semelhantes a pínulas lobuladas, $\operatorname{com} 3 \mathrm{~cm}$ de comprimento em média e ápice obtuso arredondado, quando indiferenciadas, ou verdadeiramente pinadas, linearlanceoladas, quando diferenciadas. Raque da pina semelhante a uma veia média, de curso reto, com veias laterais dicotomizadas desigualmente, duas ou mais vezes no seu curso até a margem. Pínulas membranáceas, inseridas reta ou obliquamente, por toda a extensão de suas bases de modo alterno e contíguo, terminando em ápice obtuso arredondado, com $12 \mathrm{~mm}$ de comprimento médio. Venação tênue, veia média ascendendo até próximo ao ápice, quando dicotomizam-se uma vez; veias laterais surgem da veia média alternadamente e dicotomiza-se 1-2 vezes em seu curso curvo até a margem. Seis a 11 pares de sinângios circulares dispostos paralelamente à veia média, portando de 5 a 6 (raramente 4) esporângios. Esporos monoletes ou pseudo-triletes, levemente pontuados, quasilævigate.

Descrição. Fragmentos de frondes tripinadas com no mínimo $300 \mathrm{~mm}$ de comprimento e $160 \mathrm{~mm}$ de largura. Raque principal bastante grossa, de até $20 \mathrm{~mm}$ de largura. Pinas primárias de contorno triangular/lanceolar, dispostas muito próximas entre si (por vezes tocando-se ou sobrepondo-se na base). Raques das pinas primárias igualmente grossas, de 6 a $10 \mathrm{~mm}$ de largura, retas ou levemente arqueadas em direção ao ápice da fronde, inseridas alternadamente na raque principal em ângulos entre $40^{\circ} \mathrm{e} 70^{\circ}$.

As pinas secundárias podem apresentar contornos límbicos distintos, conforme o estágio ontogenético em que se encontre. No início de seu desenvolvimento, apresentam contorno lobulado. em estágios intermediários, apresentamse pinatífidas; quando maduras, apresentam contorno verdadeiramente pinado. Nos estágios iniciais de seu desenvolvimento, as pinas assemelham-se a grandes pínulas. As pinas secundárias dispõem-se alternadamente ao longo das raques primárias, muito próximas umas das outras, em ângulos entre $50^{\circ} \mathrm{e} 90^{\circ}$, por vezes se inclinando em direção ao ápice das pinas primárias. As maiores pinas secundárias registradas tinham até $80 \mathrm{~mm}$ de comprimento, por $14 \mathrm{~mm}$ de largura e raques com até $2,1 \mathrm{~mm}$ de largura. Dada a polimorfia destas frondess, pínulas verdadeiras são vistas somente nas pinas secundárias completamente diferenciadas. As pínulas são digitiformes, de margens paralelas e encontram-se inseridas alternadamente à raque secundária por toda a extensão de suas bases. Esta inserção se dá a partir de suas nervuras principais, segundo ângulos entre $60^{\circ}$ a $80^{\circ}$. Podem alcançar até $10 \mathrm{~mm}$ de comprimento por $5 \mathrm{~mm}$, perfazendo uma razão comprimento/largura de aproximadamente 1,4 a 2,5.

A veia média das pínulas tem entre 0,3 a $0,5 \mathrm{~mm}$ de largura, são retas e indivisas até quase o ápice, onde se dividem uma única vez. Nervuras laterais ocorrem em elevada densidade (até 2 por $\mathrm{mm}$ ), divergindo de modo alterno e decorrente da veia média, mas logo curvam-se em direção à margem pinular. Em seu terço inicial, as nervuras laterais bifurcam-se e os ramos resultantes alcançam a margem da pínula em ângulos próximos a $90^{\circ}$. Os sinângios tem a forma de rosetas, com até $1,5 \mathrm{~mm}$ de diâmetro e dispõem-se próximos à extremidade distal das nervuras laterais, bastante próximos uns dos outros. Embora possam ser registrados quatro ou seis esporângios fusiformes por sinângio, o mais comum é encontrar cinco.

Discussão. Asterotheca piatnitzkyi recebe aqui uma emenda à diagnose de forma a contemplar a variação nos seus tipos de pinas e pínulas. Esta polimorfia pode ser melhor vista em sua expressão estéril típica e exclusiva, em exemplares de $P$. pedrasica (Figuras 3, 4). Propõe-se aqui Asterotheca piatnitzkyi como único nome válido para os espécimes exclusivamente férteis que reúnam as características constantes na diagnose e descrição aqui apresentadas.

Tabela 1. Medidas obtidas para A. piatnitzkyi e $P$. pedrasica. Abreviaturas: L, Largura; C, comprimento; A, ângulo; P1, pinas Primárias; P2, pinas secundárias; $\mathbf{P}$, Pínula; NS, nervuras laterais (secundárias); S, sinângios; $\boldsymbol{\varnothing}$, diâmetro; E, esporângios.

Tabela 1. Measurements of $A$. piatnitzkyi and $P$. pedrasica. Abreviations: L, width; $\mathbf{C}$, length; $\mathbf{A}$, angles; $\mathbf{P 1}$, primary pinnae; $\mathbf{P 2}$, secondary pinnae; $\mathbf{P}$, pinnulae; $\mathbf{N S}$, lateral (secondary) veins; $\mathbf{S}$, sinangia; $\varnothing$, diameter; E, esporangia.

\begin{tabular}{|c|c|c|c|}
\hline \multicolumn{2}{|c|}{ Parâmetros } & A. piatnitzkyi & P. pedrasica \\
\hline \multirow{5}{*}{ Frondes } & $\mathbf{L}$ & $>52$ & $>160$ \\
\hline & C & $>50$ & $>245$ \\
\hline & L Raque & $<20$ & $<11$ \\
\hline & A P1 & $45^{\circ}$ a $50^{\circ}$ & $60^{\circ}-70^{\circ}$ \\
\hline & P1/100 mm & 2 & 2 \\
\hline \multirow{5}{*}{ P1 } & LP1 & $<200$ & $<200$ \\
\hline & C & $<90$ & $<125$ \\
\hline & L Raque & 6,0 a 10 & 6 \\
\hline & A P2 & $50^{\circ}-90^{\circ}$ & $45^{\circ}-90^{\circ}$ \\
\hline & $\mathrm{P} 2 / 50 \mathrm{~mm}$ & $<6$ & $<6$ \\
\hline \multirow{5}{*}{ P2 } & $\mathbf{L}$ & $<15$ & $<12$ \\
\hline & C & $<80$ & $<80$ \\
\hline & L Raque & $1,0-2,1$ & 0,4 \\
\hline & A PI & $60^{\circ}-90^{\circ}$ & $50^{\circ}-90^{\circ}$ \\
\hline & $\mathrm{PI} / 10 \mathrm{~mm}$ & $2-3$ & $2-3$ \\
\hline \multirow{3}{*}{$\mathbf{P}$} & $\mathbf{L}$ & $2-6$ & $2,4-5,2$ \\
\hline & C & $4-10$ & $4,0-12,0$ \\
\hline & C/L & $1,5-2,5$ & $1,6-2,3$ \\
\hline \multirow{3}{*}{ Venação } & A NS & $30^{\circ}-35^{\circ}$ & $20^{\circ}-30^{\circ}$ \\
\hline & $\mathrm{N}^{\circ} \mathrm{NS}$ & $<12$ & $<12$ \\
\hline & $\mathrm{NS} / \mathrm{mm}$ & 1 & 1 \\
\hline \multirow{3}{*}{$\begin{array}{l}\text { Estruturas } \\
\text { reprodutivas }\end{array}$} & $\mathrm{N}^{\circ} \mathrm{S}$ & $3-11$ & - \\
\hline & $\varnothing \mathrm{s} / \mathrm{mm}$ & $1,0-1,5$ & - \\
\hline & $\mathbf{N}^{\circ} \mathrm{E} / \mathrm{S}$ & $4-6$ & - \\
\hline
\end{tabular}


As pinas em vias de diferenciação de A. piatnitzkyi assemelham-se a P. buttsii White (Gastaldo, 1984), forma lobulada encontrada em estratos do Pennsylvaniano Inferior do Alabama e do Tennessee. Porém, de acordo com o esquema de venações complexas proposto por Gastaldo \& Matten (1978), A. piatnitzkyi apresenta venação do tipo P. lamuriana Heer, enquanto que $P$. buttsii apresenta o tipo descrito para P. pseudovestita White. Além disso, tratam-se de espécimes em diferente estágio de vida, sendo um fértil e outro estéril, inviabilizando comparações mais detalhadas. Asterotheca oreopteridia Schlotheim e A. orientalis (Schenk.) Pot. (Halle, 1927) são bastante semelhantes a $A$. piatnitzkyi, mas, conforme salientado por Archangelsky \& De La Sota (1960), a escassez de informações impossibilita maiores comparações.

A. piatnitzkyi Frenguelli (1953) assemelha-se muito a $A$. golondrinensis Herbst (Archangelsky et al., 1999), mas, ao contrário desta, não possui margens dentadas. Já $A$. frenguellii Archangelsky \& De La Sota (Cúneo et al., 2000), é muito similar a $A$. piatnitzkyi, embora isto não tenha sido discutido quando da criação da primeira. Há algumas discrepâncias quantitativas entre ambas, mas a maioria não é significativa. A única exceção é o comprimento pinular sensivelmente maior de $A$. frenguellii e, em função disto, uma ligeira diferença na forma das pínulas desta espécie. Considerando porém que os espécimes descritos como $A$. frenguellii não foram aqui analisados, mantêm-se por ora a distinção entre esta e A. piatnitzkyi com base nas descrições constantes na literatura recente (Cúneo et al., 2000).

\section{Grupo PTERIDOPHYLLA (Incertae sedis) \\ Morfogênero Pecopteris (Brongn.) Sternb. (emend. Vieira et al.)}

Morfoespécie-tipo. Pecopteris pennaeformis Brongniart, 1822

Pecopteris pedrasica Read (emend. Vieira et al.)

(Figuras 3 e 4; Tabela 1)

Pecopteris pedrasica. Read, Monografia XII Div. Geol. Mineral. 72-3, pl. 2, figs.6-9, 1941

Pecopteris cambuhyensis Read, Monografia XII Div. Geol. Mineral. p.74-5. pl. 3, figs. 8-9, 1941

Pecopteris pedrasica. Cazzulo-Klepzig, Pesquisas, 11:259, fig. 7a; est. 7, fig. 1; est. 8, fig. 1b, 1978

Pecopteris sp.3 Rohn \& Rösler, Bol. IG-USP, 17:70. figs. 9, 357. 1986

Pecopteris sp. II .Vieira \& Iannuzzi, Pesquisas, 27(2):60, fig. 3c, 2000

Pecopteris cf. P. pedrasica. Vieira et al., An. Acad. Br. Cienc., 76(1):123, fig. 4a,b, 2004

Plesiótipo. USNM 40612, sec. paleobot., Nat. Mus. Nat. Hist., Read, 1941:est. 2, fig. 7.

Material estudado. MP-Pb 160, 165, 168, 171, 175, 179; USP GP/3E 434, 651, 655, 656, 657, 661, 664, 668, 669, 673, 675, 678, 682, 684, 688, 690, 691, 749, 750, 753, 754, 756, 758, 759, 2092.

Emmended diagnosis. Fronds lanceolate, tripinnate and polymorphic. Frond rachises are $12 \mathrm{~mm}$ in diameter, sometimes striated. Primary pinnae are $6 \mathrm{~mm}$ in diameter, lanceolate, alternately inserted upon the main rachis at right angles and very close each other. Secondary pinna are $0.5 \mathrm{~mm}$ in diameter, alternately inserted upon primary rachis at $50^{\circ}$ a $80^{\circ}$ angles and very close each other. Undifferentiated secondary pinnae are pinnatifid or similar to lobulated pinnulae with rounded apex. Differentiated secondary pinna are true pinnate. Secondary pinnae average approximately $70 \mathrm{~mm}$ long and $12 \mathrm{~mm}$ wide. Secondary rachis of undifferentiated rachises looks like mid-vein with two or more divisions. True pinnules are inserted upon secondary pinna rachis at approximately right angles. These pinnules are very close each other and are inserted alternately upon secondary rachis by their entire basis. They average $5 \mathrm{~mm}$ in length and $2.5 \mathrm{~mm}$ in wide and have straight and parallel margins. A slightly decurrent midvein arises straight and not divided from secondary rachis, ascending to apex and dichotomizing once near them. 5 to 12 decurrent lateral veins arise alternately from the midvein, dichotomizes near the midvein and the resulting veins continue to the margin at right angles.

Diagnose emendada. Frondes polimórficas, lanceoladas, no mínimo tripinadas. Raque principal com $12 \mathrm{~mm}$ de largura média e estriada longitudinalmente. Pinas primárias lanceoladas, bastante próximas umas das outras, dispostas alternadamente em ângulo perpendicular e com largura média da raque igual a $6 \mathrm{~mm}$. Pinas secundárias dispostas alternadamente sobre as raques das pinas primárias, em ângulos de $50^{\circ}$ a $80^{\circ}$, muito próximas umas das outras e com raque igual a $0,5 \mathrm{~mm}$ de largura média. Pinas secundárias indiferenciadas são pinatífidas ou semelhantes a uma pínula lobulada com até $70 \mathrm{~mm}$ de comprimento médio e $12 \mathrm{~mm}$ de largura média. Raque secundária semelhante a uma veia média com nervuras laterais no mínimo bifurcadas. Pinas secundárias diferenciadas são verdadeiramente pinadas, com pínulas dispostas alternadamente ao longo da raque, muito próximas umas das outras, em ângulos de $50^{\circ}$ e $90^{\circ}$. Pínulas com $5 \mathrm{~mm}$ de comprimento médio por $2,5 \mathrm{~mm}$ de largura média, inseridas à raque por toda a extensão de suas bases, com margens retas e paralelas. Veia média reta e indivisa até quase o ápice, onde se divide uma única vez. De 5 a 12 veias laterais partem alternada e decorrentemente da veia média, mas inflexionando-se em direção às margens das pínulas, dividindo-se uma única vez próximo ao primeiro terço de sua extensão.

Descrição. Fragmentos de frondes tripinadas, com no mínimo $160 \mathrm{~mm}$ de comprimento e $245 \mathrm{~mm}$ de largura. Raque principal com 10,9 mm de largura máxima e estriada longitudinalmente. Pinas primárias inseridas na raque principal com ângulos entre $60^{\circ}$ e $70^{\circ}$, pinas secundárias inseridas à raque primária em ângulos de $50^{\circ}$ a $80^{\circ}$. Há, aproximadamente, 2 pinas primárias a cada $10 \mathrm{~cm}$ de raque principal e 5 ou 6 pinas secundárias a cada $5 \mathrm{~cm}$ de pina primária. Pinas primárias lanceoladas, com até $90 \mathrm{~mm}$ de largura, $125 \mathrm{~mm}$ de comprimento máximo e raque primária com até $6 \mathrm{~mm}$ de largura. Pinas secundárias com 12 mm de largura mínima, $80 \mathrm{~mm}$ de comprimento máximo, ápices arredondados, e raque secundária de até $0,4 \mathrm{~mm}$ de largura. Ocorrem aproximadamente 2 pínulas por centímetro de raque 

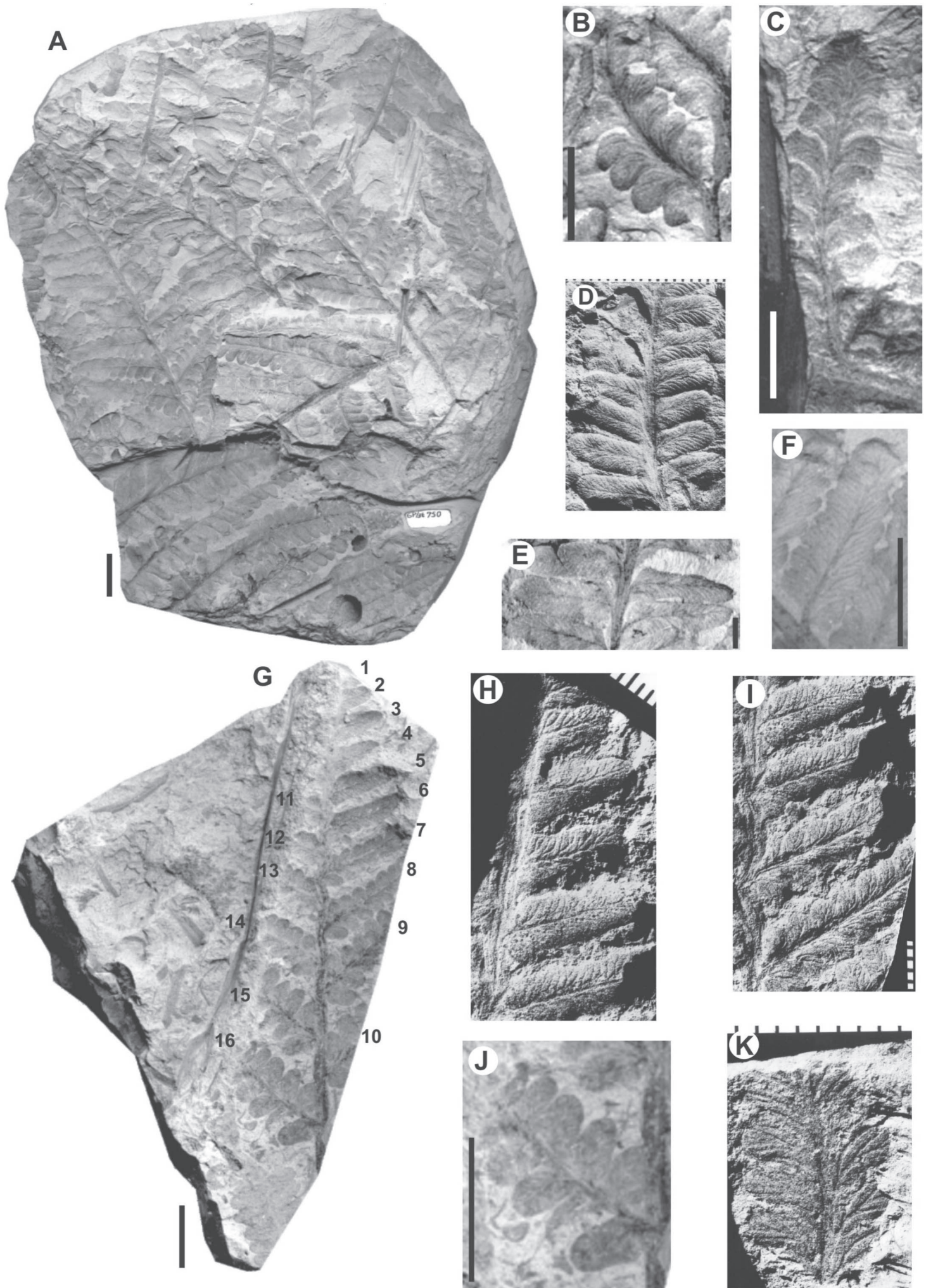

Figura 3. Pecopteris pedrasica: A, fragmento de fronde (GP/3E 750), escala $=10 \mathrm{~mm}$; B-C, pina secundária pinatífida (GP/3E 688 e 691 ), escala $=10 \mathrm{~mm}$; D, pina secundária (GP/3E 690), escala $=5 \mathrm{~mm}$; E, porção apical de uma pina primária $(\mathrm{GP} / 3 \mathrm{E} 661)$, escala $=5 \mathrm{~mm} ; \mathbf{F}$, pina de secundária (GP/3E 651), escala $=10 \mathrm{~mm} ; \mathbf{G}$, fragmento de uma pina primária (GP/3E 2094), escala $=10 \mathrm{~mm} ; \mathbf{H}$, porção apical da pina primária (vista em G 1,2,3 e 6), escala $=5 \mathrm{~mm}$; I, porção mediana da pina primária (vista em G 5,7 e 8), escala = $5 \mathrm{~mm}$; J, porção basal da pina primária (vista em G 15 e 16), escala $=10 \mathrm{~mm} ; \mathbf{K}$, pina secundária pinatífida (GP/3E 688), escala $=5 \mathrm{~mm}$.

Figure 3. Pecopteris pedrasica: A, frond fragment (GP/3E 750), scale bar $=10 \mathrm{~mm}$; B-C, pinnatifid secondary pinna detail (GP/3E 688 and 691), scale bar $=10 \mathrm{~mm}$; D, secondary pinna (GP/3E 690), scale bar $=5 \mathrm{~mm}$; E, primary pinna apical portion $(\mathrm{GP} / 3 \mathrm{E} 661)$, scale bar $=5 \mathrm{~mm}$ $\mathbf{F}$, secondary pinna (GP/3E 651), scale bar $=10 \mathrm{~mm}$; G, primary pinna fragment (GP/3E 2094), scale bar $=10 \mathrm{~mm} ; \mathbf{H}$, primary pinna apical portion (depicted in G1,2,3 and 6), scale bar $=5 \mathrm{~mm}$; I, primary pinna mid-portion (depicted in G5,7 and 8), scale bar $=5 \mathrm{~mm} ; \mathbf{J}$, primary pinna basal portion (depicted in G 15 and 16), scale bar $=10 \mathrm{~mm}$; K, a pinnatifid secondary pinna (GP/3E 688 ), scale bar $=5 \mathrm{~mm}$. 


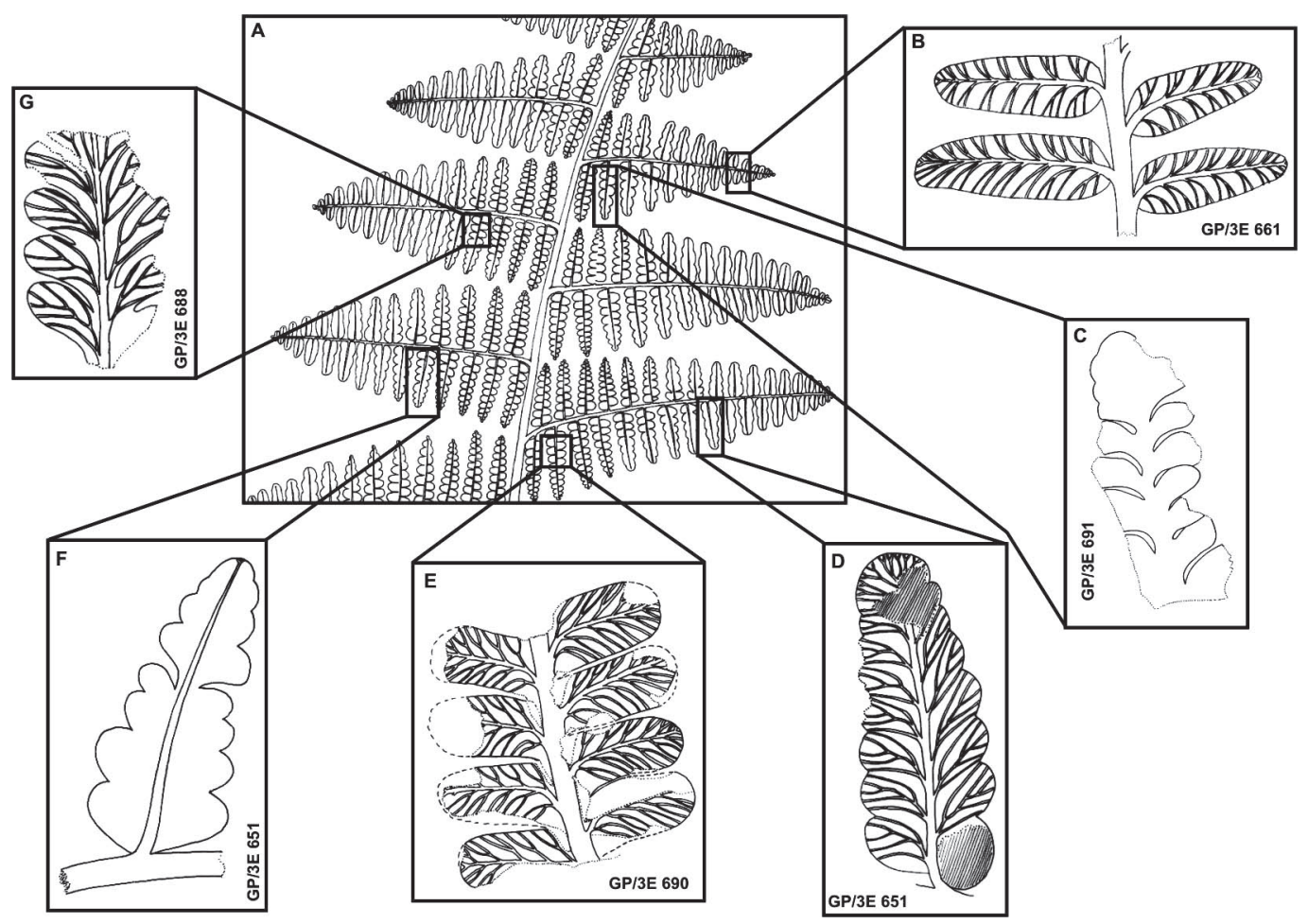

Figura 4. Pecopteris pedrasica. A, reconstituição de uma fronde baseada na associação de dois ou mais tipos de pinas e pínulas; B, pina primária com pinas secundárias semelhantes a pínulas neuropterídeas (correspondente à Figura $3 E$ ); C, D, F, G, pinas secundárias com pínulas em vias de diferenciação (correspondendo, respectivamente, a Figura 3C, F, C e B); notar o progressivo lobulamento das margens límbicas, conferindo um aspecto lobatopterídeo; E, pina secundária com pínulas já plenamente diferenciadas (correspondendo à Figura 3D), notar o padrão pecopterídeo das pínulas.

Figure 4. Pecopteris pedrasica. A, frond reconstitution based on two or more kind of pinna and pinnula association; B, primary pinnae fragment with secondary pinnae similar to neuropterid-like pinnula (corresponding to 3E figure); C, D, F, and G, secondary pinna fragments with undifferentiated pinnula (corresponding to Figure 3C, F, C, B respectively); note the increasing lobulation of limbic margins resulting in lobatopterid aspect; E, secondary pinna fragment with fully differentiated pinnula (corresponding to 3D figure), note the pecopterid pattern of pinnula.

na pina secundária, inseridas alternadamente, com ângulos entre $50^{\circ}$ e $90^{\circ}$. Pínulas medindo de 2,4 a $5,2 \mathrm{~mm}$ de largura, 4,0 a $12,4 \mathrm{~mm}$ de comprimento, perfazendo uma razão comprimento/largura de 1,6 a 2,3. As pínulas são digitiformes, de margens retas e paralelas. A venação é visível, tênue, composta por uma veia média distinta, reta e indivisa até quase o ápice, quando divide-se uma vez; veias laterais partem alternadamente da veia média, em ângulos de $20^{\circ}$ a $30^{\circ}$, inflexionando em seguida, de modo a formar arcos que resultam em ângulos retos entre a porção terminal das veias laterais e a margem das pínulas. Há cinco a seis pares nervuras secundárias por pínula, numa densidade de aproximadamente uma nervura lateral por milímetro de veia média.

Discussão. Considerando seu caráter polimórfico, P. pedrasica recebe aqui uma emenda a sua diagnose, tornando seu epíteto válido apenas para os espécimes estéreis que reúnam as características constantes na diagnose aqui apresentada. Assim, inclui os espécimes estéreis do material previamente descrito como Pecopteris sp. II (Feruglio, 1933, 1951), P. pedrasica e $P$. cambuhyensis (Read 1941, Frenguelli, 1953), A. piatnitzkyi (Archangelsky \& De La Sota, 1960), A. (P.) cambuhyensis (Rösler, 1972), P. pedrasica (Cazzulo-Klepzig, 1978), Pecopteris sp. II (Vieira \& Iannuzzi, 2000a), P. cf. P. pedrasica (Vieira et al., 2004) e P. pedrasica (Vieira, 2004).
Rohn \& Rösler (1986) descreveram uma forma pecopterídea bastante semelhante a $P$. pedrasica, mas como esta possuía pínulas maiores, classificaram-na como Pecopteris sp. 3. Considerando-se que o que foi descrito por Read (1941) como P. pedrasica é, na realidade, uma pina em vias de diferenciação, é natural que haja diferenças nas dimensões e espaçamento das pínulas desta e de Pecopteris sp. 3, uma vez que ainda estão em crescimento. De fato, significativas variações de dimensões nestas frondes polimórficas já foram notadas anteriormente (Feruglio, 1933; Frenguelli, 1953), porém, foram aqui descartadas como caráter diagnóstico para classificação em função das razões expostas acima (vide Revisão das formas descritas). Assim, é possível considerar Pecopteris sp. 3 de Rohn \& Rösler (1986) como uma pina em vias de diferenciação de P. pedrasica .

Pecopteris pedrasica é bastante semelhante também a $P$. vera Gastaldo \& Matten (1978), mas se diferencia desta pelos seus distintos padrões de venação. Por fim, há algumas formas polimórficas de pecopterídeas descritas na literatura (Corsin, 1951; Boureau, 1970; Boureau \& Doubinger, 1975) que parecem assemelhar-se aos espécimes tratados aqui, mas na falta de reproduções gráficas detalhadas, não foram comparadas. Assim, são mantidas as razões originais de Read (1941) como justificativa para a validade de P. pedrasica. 


\section{CONSIDERAÇÕES FINAIS}

A ocorrência de distintas formas e estágios de vida gerou dificuldades na definição taxonômica destas frondes, com diferentes nomes sendo atribuídos apenas à variação na forma e espécimes férteis e estéreis recebendo designações idênticas. Mesmo parecendo satisfatória, esta última alternativa desconsidera o fato de que um tipo de folhagem possa ser compartilhado por (morfo)espécies com distintas estruturas reprodutivas.

Existem inúmeros exemplos de diferentes plantas atuais e fósseis que apresentam este paralelismo vegetativo. Alguns gêneros de leguminosas, por exemplo, são nitidamente distintos entre si quanto a suas estruturas reprodutivas, mas podem apresentar folhagens virtualmente idênticas. Em plantas fósseis, tais paralelismos morfológicos são ainda mais problemáticos em termos taxonômicos, pois o mais comum é que se tenham apenas fragmentos dos indivíduos e um ou outro estágio ontogenético ou reprodutivo preservado (geralmente, estágios estéreis).

Existem também exemplos de tais paralelismos vegetativos em pecopterídeas do Neopaleozóico sul-americano. Dizengotheca waltonii Archangelsky \& De La Sota, D. branisae Arrondo e A. hemitelioides Kidston possuem estruturas reprodutivas diferentes entre si, mas compartilham o mesmíssimo padrão de folhagem (Brongniart, 1828; Kidston, 1924; Archangelsky \& De La Sota, 1960; Arrondo, 1967). Assim, torna-se impossível identificar a qual das três morfoespécies pertenceriam espécimes totalmente estéreis deste padrão de folhagem. Neste caso, dá-se o nome de $P$. hemitelioides Brongniart aos espécimes estéreis (Rodrigo \& Castaños, 1980; Vieira, 2004).

No caso específico das formas aqui estudadas, não foram ainda encontradas associadas a $P$. pedrasica estruturas reprodutivas diferentes daquelas descritas para $A$. piatnitzkyi, o que poderia sugerir uma fusão destes nomes num só. Porém, este procedimento ignoraria não só a possibilidade de paralelismos vegetativos, mas também, o histórico particular destas formas. Durante o Neopermiano, não há qualquer registro de $A$. piatnitzkyi Frenguelli nas palaeofloras da América do Sul, embora $P$. pedrasica Read e outras morfoespécies de Asterotheca sejam elementos relativamente freqüentes nas mesmas (Feruglio, 1933, 1951; Frenguelli, 1953; Archangelsky, 1958; Archangelsky \& Da La Sota, 1960; Cazzulo-Klepzig, 1978; Azcuy \& Césari, 1980; Cúneo, 1983, 1987, 1990; Read, 1941; Rösler, 1972; Rohn \& Rösler, 1986; Rohn et al., 1997a,b,c; Vieira \& Iannuzzi, 2000b; Vieira et al., 2001, 2003, 2004). Assim, propõe-se a distinção parataxonômica desta forma baseado não só na possibilidade real de que $P$. pedrasica venha a ocorrer associada a outro tipo de estrutura reprodutiva, que não aquele descrito para A. piatnitzkyi, mas também em recomendações do Código Internacional de Nomenclatura Botânica (Greuter et al., 2000; Artigos 1.2 e 11.7).

Embora tenham sido inadequadamente utilizadas como base para distinções taxonômicas, as diferentes formas apresentadas pelas pinas em questão são extremamente adequadas para o entendimento da ontogenia deste tipo de fronde. Quando no início do processo de desenvolvimento, as pinas secundárias assemelham-se a grandes pínulas neuropterídeas, com margens límbicas retas, indivisas e constritas na base (Figuras 3A,E; 4A, B). Na continuidade do processo, começam a aparecer lóbulos nas margens límbicas, conferindo o aspecto de grandes pínulas lobatopterídeas às pinas (Figuras3A,E-I; 4A, D). Após, os vales das lobulações límbicas destas pinas aprofundam-se, conferindo um aspecto pinatífido à pina (Figuras $3 \mathrm{~A}-\mathrm{C}, \mathrm{G}, \mathrm{K}$; 4A,C,F,G). Ao término do desenvolvimento, ocorre a completa individualização das pínulas com a descontinuidade límbica entre os antigos lóbulos, resultando então, em pinas verdadeiramente pinadas e pecopterídeas (Figuras 3A,D,G,J; $4 \mathrm{~A}, \mathrm{E})$. Este processo morfogenético ocorre segundo gradientes topológicos na fronde, em que as porções proximais das frondes e pinas, bem como basiscópicas das pinas, são as primeiras a se diferenciar. Esta morfogênese desenvolve-se gradualmente ao longo deste gradiente, de modo que é possível encontrar pinas secundárias com dois ou mais dos padrões citados. Assim, é possível observar uma pina secundária cuja porção distal apresenta-se ainda lobada (Figura 3C), enquanto a porção mediana apresenta-se pinatífida e a proximal pinada, ou seja, com pínulas individualizadas. Este padrão de diferenciação morfogenética de frondes difere ligeiramente daqueles já descritos para frondes fósseis lobatorpterídeas e pecopterídeas (Gastaldo \& Matten, 1978; Zodrow \& Banerjee, 1993), porém, foi descrito já para algumas frondes atuais (Hagemann, 1984).

Assim, com base nos arranjos taxonômicos aqui propostos, todo e qualquer registro de $A$. piatnitzkyi passa a

Tabela 2. Unidades litoestratigráficas, idades, ambientes e países relativos aos registros de $P$. pedrasica de acordo com: Archangelsky (1958), Archangelsky \& Da La Sota (1960), Archangelsky et al. (1999), Azcuy \& Césari (1980), Azcuy et al. (2000), Cúneo (1983, 1987, 1990), Feruglio (1933, 1951), Frenguelli (1953), Holz (1999), lannuzzi et al. (2003), Read (1941), Rohn \& Rösler (1986), Rohn et al. (1997a,b,c), Rösler (1972), Suárez-Soruco \& Díaz-Martínez (1996), Vieira (2004), Vieira et al. (2001, 2003, 2004).

Table 2. Litostratigraphic units, ages, environments and countries referring to $P$. pedrasica records. Based on references listed above.

\begin{tabular}{|c|c|c|c|c|}
\hline Formação & Bacia ou Grupo & Idade & Ambiente & País \\
\hline Libertad & Paganzo & Neocarbonífero & Transicional & Argentina \\
\hline Mojón de Hierro & Tepuel-Genoa & Eopermiano & Glácio-eustático & Argentina \\
\hline La Golondrina & La Golondrina & Eopermiano & Flúvio-lacustre & Argentina \\
\hline Rio Bonito & do Paraná & Eopermiano & Flúvio-lacustre & Brasil \\
\hline Rio do Rasto & do Paraná & Neopermiano & $\begin{array}{l}\text { Transicional } \\
\text { árido }\end{array}$ & Brasil \\
\hline Chutani & Titicaca & Neopermiano & $\begin{array}{l}\text { Transicional } \\
\text { árido }\end{array}$ & Bolívia \\
\hline
\end{tabular}


ser também um registro indireto de $P$. pedrasica. Por outro lado, a possível associação de $P$. pedrasica com outros tipos de estruturas que não o de A. piatnitzkyi durante o Neopermiano da América do Sul (dada a completa ausência desta última), justifica tais definições taxonômicas, e também falseiam a recíproca, isto é, nem todo o registro de $P$. pedrasica é, necessariamente, um registro indireto de $A$. piatnitzkyi. Com isto, P. pedrasica Read torna-se, a pecopterídea de maior distribuição estratigráfica e geográfica da América do Sul, sendo registrada desde o Carbonífero Superior até o Permiano Superior, desde o sul Argentina até o sudeste do Brasil e sul da Bolívia, bem como a pecopterídea de maior abrangência ambiental (Tabela 2).

Esta ampla distribuição espacial e temporal, permite assegurar-lhe um caráter euritópico, visto que habitaram distintos ambientes (Tabela 2) e enfrentaram significativas mudanças climáticas ao longo do Neopaleozóico (Scotese \& Barret, 1990; Ziegler, 1990; Golonka \& Ford, 2000; Fluteau et al., 2001; Rees et al., 2002).

\section{AGRADECIMENTOS}

Nossos sinceros agradecimentos a L. F. Lopes (UFRGS), pela sua dedicação na obtenção de material fotográfico de alta qualidade. Por permitir e viabilizar o estudo dos espécimes depositados em coleções sob suas responsabilidades, as seguintes pessoas foram fundamentais no desenvolvimento deste trabalho: O. Rösler, T. Fairchild e I. Cassab (USP), Dr. Hugo A. Carrizo (LIL) e Dr. Carlos Azcuy (UBA). Agradecemos também a. E. Sellers e T. J. Dutro (USGS), por localizar o material original descrito por C. B. Read. Este trabalho foi desenvolvido com auxílio financeiro do CNPq, sob forma de bolsa de doutorado (CELV) e pesquisador (RI). Esta é uma contribuição do Centro de Investigações do Gondwana (CIGO) ao projeto IGCP 471.

\section{REFERÊNCIAS}

Archangelsky, S. 1958. Estudio geológico y paleontológico del Bajo de La Leona (Santa Cruz). Acta Geolica Lilloana, 2:5-135.

Archangelsky, S. \& De La Sota, E. 1960. Contribuición al conocimiento de las filices permicas de patagonia extraandina. Acta Geologica Lilloana, 3:85-126.

Archangelsky, S.; Césari, S. \& Cúneo, N. R. 1999. Revisión de Asterotheca golondrinensis Herbst, helecho pérmico de Patagonia, Argentina. Buenos Aires, Associación Paleontológica de Argentina, p. 23-26 (Publicación Especial 6).

Arrondo, O. 1967. Dizeugotheca branisae n. sp. nueva filice eusporangiada del Pérmico inferior, Bolívia. Revista del Museo de La Plata (nueva serie Paleontología), 5:55-61.

Azcuy, C.L. \& Césari, S.N. 1980. Primeros estudios sobre plantas fósiles de la Formación Libertad, Provincia de La Rioja, Argentina. Boletim IG-USP, 11:49-55.

Azcuy, C.L.; Carrizo, H.A. \& Caminos, R. 2000. Carbonífero y Pérmico de las Sierras Pampeanas, Famatina, Precordillera, Cordillera Frontal y bloque de San Rafael. In: R. Caminos (ed.) Geología Argentina, Instituto de Geologia y Recursos Minerales, p. 261-318.

Boureau, E. 1970. Traité de Paleobotanique: Filicophyta. Tome. IV, Fasc. 1, Paris, Masson et Cie., 519 p.
Boureau, E. \& Doubunguer, J. 1975. Traité de Paleobotanique: Filicophyta. Tome. IV. Fasc. 2. Pteridophylla. Paris, Masson et Cie., 768 p.

Brongniart, A. 1828. Prodrome d'une Histoire des Végétaux Fossiles. Dictionnaire des Sciences Naturelles, 57:16-212.

Cazzulo-Klepzig, M. 1978. Estudo da tafoflórula do Membro Morro Pelado na sua localidade-tipo. Pesquisas, 11:225-303.

Corda, A.J. 1845. Flora Protogaea. Beiträge zur Flora der Vorwelt. Berlin, S. Calvary and Co., 128 p.

Corsin, P. 1951. Pécoptéridés. In: P. Corsin (ed.) Etudes des Gites Mineraux de la France. Bassin Houiller de la Sarre et de la Lorraine, Service Geologique de France, p. 175-370.

Cúneo, R. 1983. Paleoecología de microsecuencias plantíferas del Grupo Rio Genoa, Pérmico de Chubut, Argentina. Ameghiniana, 20(1-2):111-131.

Cúneo, R. 1987. Fitopaleoecología de la Formación Rio Genoa em la localidad Lomas Chatas, Pérmico de Chubut, Argentina. Ameghiniana, 24(1-2):3-15.

Cúneo, R. 1990. La tafoflora de la Formación Mojon de Hierro (Grupo Tepuel) en la localidad Arroyo Garrido, Paleozóico superior, Província de Chubut. Ameghiniana, 27(3-4):225-238.

Cúneo, N.R.; Archangelsky, S. \& Césari, S.N. 2000. Asterotheca frenguellii (Archangelsky \& De La Sota) nov. comb., helecho pérmico de Patagonia, Argentina. Ameghiniana, 37(3):363-367.

Diggle, P.K. 1999. Heteroblasty and the evolution of flowering phenologies. International Journal of Plant Science, 160(S6):123134.

Feruglio, E. 1933. Fossili liassici della valle del rio Genua (Patagonia). Giornale di Geologia, Annali Revista di Museo Geologico di Bologna, 9:1-54.

Feruglio, E. 1951. Su alcune piante del Gondwana inferiore della Patagonia. Monografia di Instituto Geolico de la Universitat di Torino, I: $1-34$.

Fluteau, F.; Besse, J.; Broutin, J. \& Ramstein, G. 2001. The Late Permian climate. What can be inferred from climate modelling concerning Pangea scenarios and Hercynian range altitude? Paleogeography, Paleoclimatology, Paleoecology, 167:39-71.

Frenguelli, J. 1953. Las pecopteroideas del pérmico del Chubut. Notas del Museo Eva Perón, 16(99):287-296.

Gastaldo, R.A. \& Matten, L.C. 1978. Studies on north american pecopterids. I. Pecopteris vera n. sp. from the middle pennsylvanian of southern Illinois. Palaeontographica, Abt.B, 165(1-3):43-52.

Gastaldo, R.A. 1984. Studies on north american pecopterids. III. Pecopteris buttsii D. White from the Early Pennsylvanian of Alabama. Journal of Paleontology, 58(1):63-77.

Goliber, T.; Kessler, S.; Chen, J.J.; Bharathan, G. \& Sinha, N. 1999. Genetic, molecular, and morphological analyses of compound leaf development. Current Topics on Developmental Biology, 43:259-290.

Golonka, J. \& Ford, D. 2000. Pangean (Late Carboniferous-Middle Jurassic) paleoenvironment and lithofacies. Paleogeography, Paleoclimatology, Paleoecology, 161(1-2):1-34.

Greuter, W.; McNeill, J.; Barrie, F.R.; Burdet, H.-M.; Demoulin, V.; Filgueiras, T.S.; Nicolson, D.H.; Silva, P.C.; Skog, J.E.; Trehane, P.; Turland, N.J. \& Hawksworth, D.L. 2000. International Code of Botanical Nomenclature (St. Louis Code). Regnum Vegetabile, 138:1-474

Guerrant Jr., E.O. 1988. Heterochrony in plants: the intersection of evolution ecology and ontogeny. In: M.L. McKinney (ed.) Heterochrony in Evolution. A multidisciplinary approach Topics in Geobiology, Plenum Press, p. 111-133. 
Hagemann, W. 1984. Morphological aspects of leaf development in ferns and angiosperms. In: R.A. White \& W.C. Dickson (eds.) Contemporary Problems in Plant Anatomy, Academic Press, p. 301-349.

Halle, T. 1927. Palaeozoic plants from Central Shansi. Paleontologia Sinica, Ser. A, 2:1-316.

Kerstetter, R. A. \& Poethig, R. S. 1998. The specification of leaf identity during shoot development. Annual Review of Cellular Development Biology, 14:373-398.

Kidston, R. 1924. Fossil plants of the Carboniferous rocks of Great Britain. London, Geological Survey Great Britain, 522 p. (Memoir Paleontology II, parts 1-6).

Read, C.B. 1941. Plantas fósseis do Neo-paleozóico do Paraná e Santa Catarina. Rio de Janeiro, Ministério da Agricultura, Divisão de Geologia e Mineralogia, 102 p. (Monografia 12).

Rees, P.M.; Ziegler, A.M.; Gibbs, M.T.; Kutzbach, J.E.; Behling, P.J. \& Rowley, D.B. 2002. Permian phytogeographic patterns and climate data/model comparisons. Journal of Geology, 110:1-31.

Rodrigo, L.A. \& Castaños, A. 1980. Sinopsis estratigráfica de Bolivia. I Paleozóico. La Paz, Academia Nacional de Ciências de Bolivia, 146 p. (Publicación Special 1).

Rohn, R.; Pennatti, J.R.R.; Iannuzzi, R.; Rösler, O.; Czajkowiski, S.; Cavalheiro, M.C.T.; Mendonça, E. \& Ricardi-Blanco, F.T. 1997a. Ocorrências de Macrofitofósseis nas Formações Teresina e Rio do Rasto (Permiano superior da Bacia do Paraná) em SC, BR. Revista da Universidade de Guarulhos, Geociências, 2( ${ }^{\circ}$ especial):49-57.

Rohn, R.; Rösler, O.; Pennatti, J.R.R.; Czajkowiski, S.; Iannuzzi, R.; Mendonça, E.; Ferreira, A.R.; Pereira, S.C.A. \& Quitério, L. 1997b. Ocorrências de Macrofitofósseis nas Formações Teresina e Rio do Rasto (Permiano superior da Bacia do Paraná) na Porção Meridional do Estado do Paraná, BR. Revista da Universidade de Guarulhos, Geociências, 2(nº especial):58-68.

Rohn, R.; Rösler, O.; Pennatti, J.R.R.; Davies, H-P. K. \& Cavalheiro, M. C. T. 1997c. Ocorrências de macrofitofósseis nas Formações Teresina e Rio do Rasto (Permiano superior da Bacia do Paraná) na região de Cândido de Abreu-Reserva, Estado do Paraná, Brasil. Revista da Universidade de Guarulhos, Geociências, 2:69-75.

Rohn, R. \& Rösler, O. 1986. Pteridófilas pecopteróides da Formação Rio do Rasto no Estado do Paraná e da Formação Estrada Nova no estado de São Paulo (Bacia do Paraná, Permiano Superior). Boletim IG-USP, 17:57-76.

Rösler, O. 1972. Flora da Formação Rio Bonito do estado do Paraná. Programa de Pós-Graduação em Geociências, Universidade de São Paulo, Tese de Doutorado, 130 p.

Rösler, O. 1978. The Brazilian Eogondwanic Floral Succession. Boletim IG-USP, 9:85-90.
Scotese, C.R. \& Barrett, S.F. 1990. Gondwana's movement over the South Pole during the Palaeozoic: evidence from lithological indicators of climate. In: W.S. Mckerrow \& C.R. Scotese (eds.) Paleozoic paleogeography and biogeography, Geological Society of London, p. 75-86 (Memoir, 12).

Suarez-Soruco, R. \& Díaz-Martínez, E. 1996. Léxico estratigráfico de Bolivia. Revista Técnica del YPFB, 17(1-2):1-227.

Tsukaya, H. 2002. The leaf index: heteroblasty, natural variation, and the genetic control of polar processes of leaf expansion. Plant And Cell Physiology, 43(4):372-378.

Vieira, C.E.L. 2004. Taxonomia, paleoecologia e paleofitogeografia de frondes pecopterídeas do Neopaleozóico da América do Sul. Programa de Pós-graduação em Geociências, Universidade Federal do Rio Grande do Sul, Tese de Doutorado, 249 p.

Vieira, C.E.L. \& Iannuzzi, R. 2000a. Presença de Pecopteris e Asterotheca no Afloramento do Morro do Papaléo, município de Mariana Pimentel, Rio Grande do Sul (Formação Rio Bonito, Eopermiano da Bacia do Paraná). Pesquisas, 27(2):49-64.

Vieira, C.E.L. \& Iannuzzi, R. 2000b. Presença de Asterotheca sp. no Permiano Superior da Bacia do Paraná (Membro Serrinha, Formação Rio do Rasto). Revista da Universidade de Guarulhos, Geociências, 5:33-36.

Vieira, C.E.L.; Iannuzzi, R.; Guerra-Sommer, M. 2001. Nova forma de pecopterídea no afloramento do Morro do Papaléo, município de Mariana Pimentel, Rio Grande do Sul (Formação Rio Bonito, Permiano Inferior da Bacia do Paraná). In: CONGRESSO BRASILEIRO DE PALEONTOLOGIA, 17, 2001. Boletim de Resumos, Acre, UFAC, p. 67.

Vieira, C.E.L.; Iannuzzi, R. \& Guerra-Sommer, M. 2003. Primeiro registro de frondes férteis na Formação Pedra de Fogo, Neopermiano da Bacia do Parnaíba. CONGRESSO BRASILEIRO DE PALEONTOLOGIA, 18, 2003. Boletim de Resumos, Brasília, UnB, p. 313.

Vieira, C.E.L.; Iannuzzi, R.; Guerra-Sommer, M.; Díaz-Martínez, E. \& Grader, G. W. 2004. Permian plants from the Chutani Formation (Titicaca Group, Northern Altiplano of Bolívia): genera Pecopteris and Asterotheca. Anais da Academia Brasileira de Ciências, 76(1):1-12.

Ziegler, A.M. 1990. Phytogeographic patterns and continental configuration during the Permian period. In: W.S. Mckerrow \& C.R. Scotese (eds.) Paleozoic paleogeography and biogeography, Geological Society of London, p. 363-379 (Memoir, 12).

Zodrow, E.L. \& Banerjee, S.K. 1993. Modeling the lobatopterid frond (tree fern, carboniferous). Comptes Rendus, XII ICC-P, 2:159-172.

Received in November, 2006; accepted in June, 2007 